\section{L'érythroferrone, un régulateur érythroïde du métabolisme du fer}

Léon Kautz
Department of Medicine ; David Geffen School of Medicine, University of California, Los Angeles, UCLA, department of Medicine, 10833 LeConte avenue, CHS37-131, CA90095 Los Angeles, CA, États-Unis. Ikautz@mednet.ucla.edu

poïèse, la modulation de la production d'hepcidine et la disponibilité en fer était mal caractérisé. II était communément admis qu'un facteur inconnu, historiquement désigné sous le nom de « régulateur érythroïde » [3], contrôlait ce processus. L'érythropoïèse est régulée principalement par l'érythropoïétine ( $\varepsilon P 0)$, un facteur de croissance indispensable à la survie et à la prolifération des précurseurs érythroïdes. Dans des conditions

particulières telles qu'une anémie ou mentées afin de normaliser rapidement le nombre de globules rouges et la capacité de transport de l'oxygène. Pour faire face à ce besoin accru en fer, la production d'hepcidine diminue de manière à stabiliser la ferroportine à la surface des cellules qui l'expriment, ce qui conduit à l'augmentation de l'absorption du fer alimentaire au niveau du duodénum et du relargage du fer par les macrophages et les hépatocytes. Jusqu'à récemment, le lien entre l'augmentation de l'érythro-

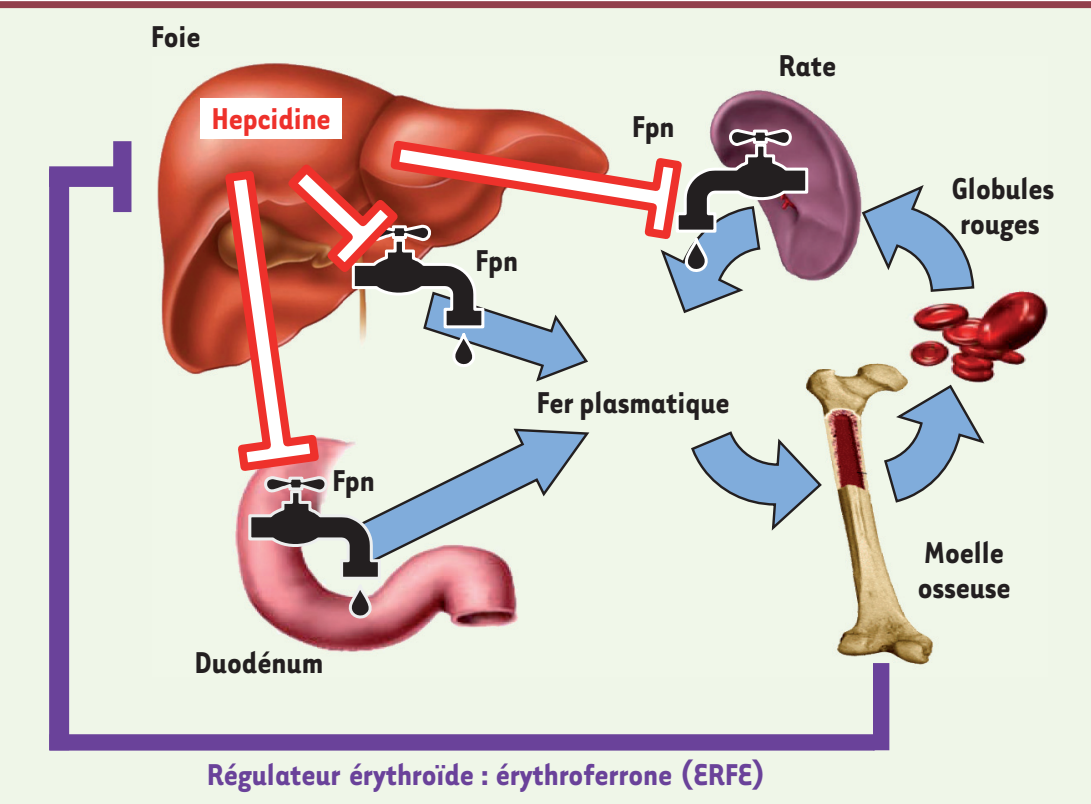

Figure 1. L'hepcidine coordonne la disponibilité en fer pour l'érythropoïèse. L'hepcidine contrôle l'entrée du fer dans la circulation en fixant la ferroportine (Fpn) à la surface des entérocytes et des macrophages dans le foie et la rate. Ceci provoque la dégradation de la ferroportine, bloquant le fer dans ces cellules. En cas de besoin en fer accru lors d'une forte activité érythropoïétique, les précurseurs érythroïdes produisent un ou des facteurs appelé(s) régulateur(s) érythroïde(s), dont la fonction est de réprimer l'expression de l'hepcidine dans le foie afin d'augmenter la disponibilité en fer pour la moelle osseuse. Nos travaux décrivent l'identification d'un nouveau régulateur érythroïde, l'érythroferrone ( $\varepsilon R F \varepsilon$ ) (๔ cette figure a été préalablement publiée dans Blood [9]). 
une l'hypoxie, le taux d'EPO augmente conjointement à la diminution de l'expression de l'hepcidine. Les données de la littérature suggéraient que la régulation de l'hepcidine n'était pas directement dictée par l'EPO, mais impliquait un facteur rapidement produit par les précurseurs érythroïdes de la moelle osseuse, puis sécrété dans la circulation pour réprimer la production d'hepcidine afin d'augmenter la quantité de fer disponible pour la synthèse de nouveaux globules rouges par la moelle osseuse [4-7].

\section{Les troubles du métabolisme du fer} et de l'érythropoïèse

Les régulateurs érythroïdes peuvent causer des complications cliniques sévères chez les patients atteints de thalassémie ou d'anémie dysérythropoïétique congénitale. Ces pathologies, qui ont en commun une érythropoï̀se inefficace, s'accompagnent d'un niveau d'EPO sérique élevé et d'une production massive de précurseurs érythroïdes qui subissent une apoptose accélérée au stade d'érythroblaste. Ceci a pour conséquence une importante diminution de la production de l'hepcidine [8]. La suppression pathologique de la synthèse de l'hepcidine conduit à une hyperabsorption du fer alimentaire et à une surcharge en fer multiviscérale délétère, indépendante du fait que les patients soient transfusés ou non. Le régulateur érythroïde responsable de ces effets exercerait donc son action inhibitrice sur l'expression de l'hepcidine non seulement dans la phase de récupération après une hémorragie, mais aussi dans les pathologies associées à une érythropoïèse inefficace, ces conditions ayant en commun un niveau élevé d'EPO et l'expansion des compartiments érythroïdes. Bien que plusieurs candidats aient été proposés comme régulateurs érythroïdes potentiels (GDF15 [growth differentiation factor 15], TWSGl [twisted gastrulation BMP signaling modulator 1] etc.), leur importance physiologique dans cette régulation reste à confirmer [9].

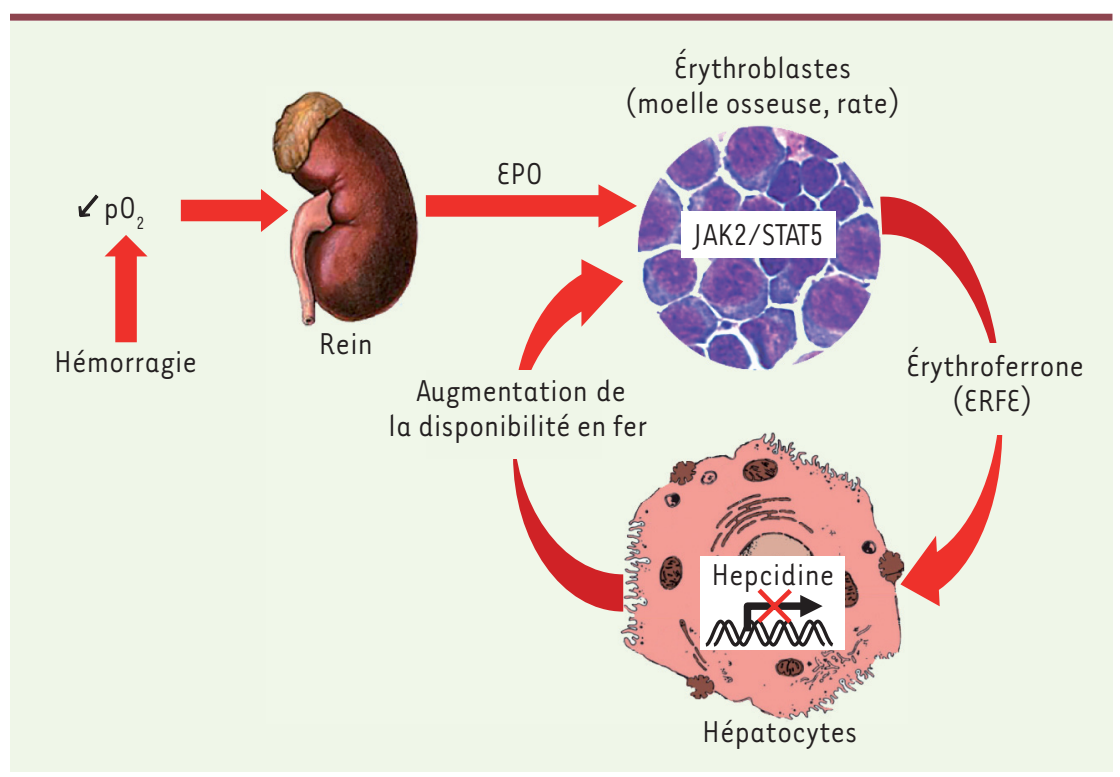

Figure 2. Mécanisme d'action de l'érythroferrone. Après une hémorragie ou une hémolyse, la diminution de la concentration en oxygène conduit à la production d'EPO par le rein. L'EPO se fixe à son récepteur à la surface des précurseurs érythroïdes dans la moelle osseuse et la rate et stimule la voie de signalisation JAK2/STAT5 et la production rapide d'érythroferrone. ERFE est ensuite sécrétée dans la circulation et agit directement sur les hepatocytes pour réprimer l'expression de l'hepcidine et ainsi augmenter la disponibilité en fer pour la synthèse de nouveaux globules rouges. Le récepteur d'ERFE et la voie de signalisation menant à la diminution de l'hepcidine sont en cours d'identification (๔ cette figure a été préalablement publiée dans Blood [9]).

L'érythroferrone, un nouveau régulateur érythroïde de l'hepcidine Nous avons récemment identifié un nouveau facteur: l'érythroferrone (ERFE) - une hormone appartenant à la famille de protéines Clq/TNF (tumor necrosis factor) - [10], qui pourrait être le régulateur érythroïde recherché de longue date (Figure 2). En réponse à I'EPO, la synthèse d'ERFE est rapidement induite dans les précurseurs érythroïdes de la moelle osseuse et de la rate via la voie de signalisation JAK2/STAT5. Nous avons montré que les souris invalidées pour ERFE sont incapables de réprimer l'expression de l'hepcidine après phlébotomie ou administration d'EPO. ERFE est donc absolument indispensable pour diminuer rapidement l'expression de I'hepcidine lors d'une demande accrue de l'érythropoïèse. Ces souris $\varepsilon r f e^{-/-}$ présentent des paramètres hématologiques normaux, suggérant qu'ERFE n'est pas un acteur majeur de l'érythropoïèse en conditions physiologiques normales, mais que son rôle serait restreint aux situations d'érythropoïèse de stress. Á noter cependant que lors de la phase de croissance rapide et d'expansion érythroïde chez des souris juvéniles (entre 3 et 6 semaines) dépourvues d'ERFE, une érythropoïèse active est suffisante pour occasionner une légère anémie transitoire chez ces animaux. In vivo, l'injection directe de la protéine ERFE, ou sa surexpression à l'aide d'un vecteur lentiviral, a confirmé son action suppressive sur l'expression de I'hepcidine. De plus, l'expression de I'ARN messager de l'hepcidine est diminuée de façon très significative lorsque des hépatocytes primaires de souris sont traités par de l'érythroferrone recombinante. Bien que ni le récepteur d'ERFE ni la voie de signalisation qu'il induit ne soient actuellement identifiés, nos données suggèrent qu'ERFE agirait directement sur les hépatocytes pour réprimer la transcription de l'hepcidine. Nos travaux révèlent également qu'દRFદ pourrait jouer un rôle important dans la bêta thalassémie. 
Dans le modèle murin de cette pathologie $\left(H b b^{\text {th3/th3 }}\right)$, le taux d'ARN messager d'Erfe est très significativement augmenté dans la moelle osseuse et la rate. De façon intéressante, l'invalidation du gène $\varepsilon r f e$ chez les souris thalassémiques $\mathrm{Hb}^{\text {th3/th3 }}$ conduit au rétablissement du niveau physiologique d'hepcidine et à une diminution significative de la sidérémie et de la surcharge en fer dans le foie. ERFE pourrait donc également agir comme répresseur de l'hepcidine dans certaines pathologies humaines. Une étude plus approfondie chez les patients atteints de bêta-thalassémie ou d'autres formes d'anémie héréditaire est désormais nécessaire afin de confirmer que l'érythroferrone est le régulateur érythroïde responsable de la diminution de l'hepcidine et de la surcharge martiale chez ces patients.

\section{Perspectives}

Nos travaux ont mis en évidence le rôle fondamental de l'érythroferrone, un nouveau facteur érythroïde, dans la régulation physiopathologique de l'hepcidine lors d'une érythropoï̀se accrue. Notre équipe développe actuellement une méthode de dosage de l'érythoferrrone afin d'étudier son rôle chez I'homme. Enfin, nos travaux montrent qu'ERFE est une nouvelle cible thérapeutique. En l'inhibant, on pourrait augmenter la production d'hepcidine chez les patients atteints d'anémie héréditaire avec surcharge en fer. Au contraire, en l'administrant à des patients présentant une anémie associée à une maladie inflammatoire, une affection rénale chronique ou un cancer, on pourrait corriger le déficit en fer lié à un excès d'hepcidine. $\diamond$

Erythroferrone, an erythroid regulator of iron metabolism

\section{REMERCIEMENTS}

Aux Docteurs Tomas Ganz et Elizabeta Nemeth pour leur mentorat; aux Docteurs Anaïs Briot, Hélène Coppin, Marie-Paule Roth et Bertrand Grenier pour leur aide dans la préparation de ce manuscrit.

\section{LIENS D'INTÉRÊT}

L'auteur déclare n'avoir aucun lien d'intérêt concernant les données publiées dans cet article.

\section{RÉFÉRENCES}

1. Ganz T, Nemeth $\varepsilon$. Hepcidin and disorders of iron metabolism. Annu Rev Med $2011 ; 62$ : 347-60.

2. Qiao $B$, Sugianto $P$, Fung $\varepsilon$, et al. Hepcidin-induced endocytosis of ferroportin is dependent on ferroportin ubiquitination. Cell Metab 2012 ; 15 : 918-24.

3. Finch C. Regulators of iron balance in humans. Blood $1994 ; 84:$ 1697-702.

4. Ashby DR, Gale DP, Busbridge M, et al. Erythropoietin administration in humans causes a marked and prolonged reduction in circulating hepcidin. Haematologica 2010 ; 95 : 505-8.

5. Mastrogiannaki M, Matak P, Mathieu JRR, et al. Hepatic hypoxia-inducible factor-2 down-regulates hepcidin expression in mice through an erythropoietinmediated increase in erythropoiesis. Haematologica $2012 ; 97: 827-34$.

6. Pak M, Lopez MA, Gabayan V, et al. Suppression of hepcidin during anemia requires erythropoietic activity. Blood $2006 ; 108: 3730-5$.

7. Vokurka M, Krijt J, Sulc K, Necas $\varepsilon$. Hepcidin mRNA levels in mouse liver respond to inhibition of erythropoiesis. Physiol Res 2006 ; 55 : 667-74.

8. Origa R, Galanello R, Ganz T, et al. Liver iron concentrations and urinary hepcidin in betathalassemia. Haematologica 2007 ; 92 : 583-8.

9. Kautz L, Nemeth $\varepsilon$. Molecular liaisons between erythropoiesis and iron metabolism. Blood 2014 ; $124: 479-82$.

10. Kautz L, Jung G, Valore $E V$, et al. Identification of erythroferrone as an erythroid regulator of iron metabolism. Nat Genet $2014 ; 46: 678-84$.

\section{NOUVELLE}

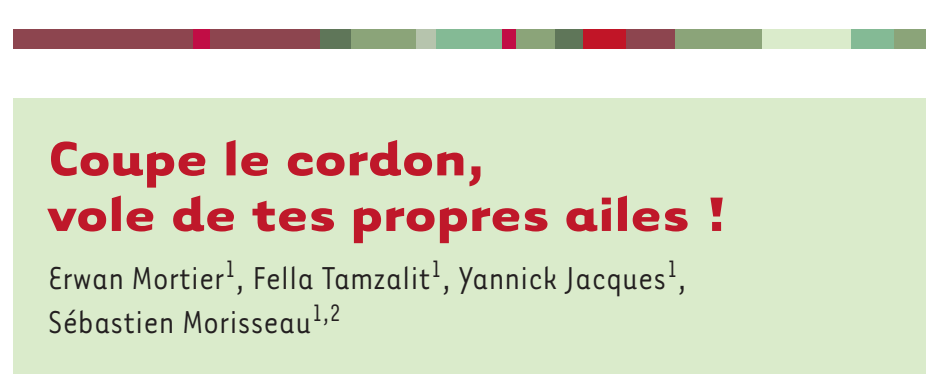

\author{
${ }^{1}$ Centre de recherche en cancérologie, Inserm UMR 892, \\ CNRS 6299, université de Nantes, 8, quai Moncousu, \\ BP70721, F-44007, Nantes, France; \\ 2 centre hospitalier universitaire, F-44093, Nantes, France. \\ erwan.mortier@inserm.fr
}

\section{L'interleukine IL-15, une cytokine au mode d'action particulier}

Les cytokines sont des messagers protéiques clés impliqués dans de nombreuses fonctions cellulaires, comme la différenciation, la prolifération, l'activation, la survie. Parmi ces cytokines, l'interleukine-15 (IL-15) est une cytokine hématopoïétique pléiotrope, inductrice physiologique des cellules immunitaires innées (NK [natural killer], NK-T et T $\gamma \delta$ ). Dans le cadre de l'immunité adaptative, le rôle de l'IL-15 est primordial dans les étapes précoces de l'activation cellulaire $T$, ainsi que pour la survie des lymphocytes T CD8 mémoires. Elle est par conséquent une cytokine importante dans la surveillance immunitaire antitumorale $[1,2]$. Les déficits dans la signalisation de I'IL-15 affectent la survie et la fonction des cellules T CD8 mémoires et NK. À l'inverse, l'excès d'IL-15 peut être impliqué dans la prolifération non contrôlée de cellules tumorales et dans certaines pathologies inflammatoires. La régulation du signal IL-15 des lymphocytes est donc critique, à la fois pour une réponse immunitaire cytolytique optimale, et pour la prévention d'un désordre lymphocytaire malin ou inflammatoire.

Identifiée en 1994 [3], I'IL-15 est structurellement proche de I'IL-2 sans que les deux cytokines ne possèdent d'homologie au niveau de leur séquence primaire. Ces deux molécules partagent des activités biologiques similaires dans le système immunitaire. Cette redondance fonctionnelle s'explique par le fait que leurs récepteurs partagent des chaînes réceptrices transductrices communes, les chaînes IL-2/15R (CD122) 